\title{
Towards Renormalizing Group Field Theory
}

\author{
Vincent Rivasseau \\ Laboratoire de Physique Théorique, CNRS UMR 8627, \\ Université Paris XI, F-91405 Orsay Cedex, France
}

March 11, 2011

\begin{abstract}
We review some aspects of non commutative quantum field theory and group field theory, in particular recent progress on the systematic study of the scaling and renormalization properties of group field theory. We thank G. Zoupanos and the organizers of the Corfu 2010 Workshop on Noncommutative Field Theory and Gravity for encouraging us to write this review.
\end{abstract}

LPT-20XX-xx

MSC: 81T08, Pacs numbers: 11.10.Cd, 11.10.Ef

Key words: Group field theory, renormalization expansion.

\section{Introduction}

Renormalization has been the soul and driving force of quantum field theory, from the early computations of the Lamb shift and of the electron anomalous magnetic moment in quantum electrodynamics to the golden era of the 70 's, when renormalization of the non-Abelian gauge theories became the key to their successful adoption as theories of the electroweak and strong interactions.

At the same time Wilson and followers understood the true meaning of renormalization. They explained it no longer as a mysterious hiding of infinities under the rug but simply as the natural change of the laws of physics 
under change of the observation scale. This brilliant step immediately lead to progress in other domains of physics. It became known under the (somewhat misleading) name of the renormalization group.

In spite of these achievements, many physicists still dream of a fundamental theory of physics that would be free from any infinities right from the start. It would have to include gravity, the "rebellious interaction". But since gravity is not perturbatively renormalizable in the naive sense, the mainstream proposal is to include it into a framework such as string theory which includes new unobserved symmetries (in particular supersymmetry) and which would make every computation finite.

It is also often suggested that absence of infinities would mean that there is a fundamental scale, eg the Planck scale, beyond which we could probe nothing, not just because of temporary experimental limits, but because nothing would exist. In this view physics and the flow of the renormalization group would not exist beyond that scale.

But we might even prefer if quantum gravity after all was just like the rest of the standard model. In particular we would like if it has perturbative divergences of a renormalizable type like electroweak and chromo quantum dynamics. These divergences could then drive an interesting renormalization group flow upstream to the Planck scale (or "before" the big bang), just like the QCD flow drives the asymptotically free physics of quarks and gluons upstream to the hadronic scale. These features could appear within a suitably enlarged and reinterpreted quantum field theory formalism. In order to find such a formalism, we should adopt a guiding principle. We share with the loop quantum gravity community the feeling that the most appealing such principle is background independence, the one principle that lead to the invention of general relativity in the first place [1]. A most natural and promising quantum field theory formalism based on these criteria is group field theory (GFT). All these considerations lead us to propose the systematic study of the scaling and renormalization properties of group field theory ${ }^{1}$.

The main encouragement which lead to such a program came from the "happy end" story of a simpler quantum field theory, the $\phi_{4}^{\star 4}$ theory on the non commutative Moyal space. For a while it was dubbed non renormalizable because of the so-called ultraviolet-infrared mixing problem. Ultimately it turned out to become renormalizable and even asymptotically safe under

\footnotetext{
${ }^{1}$ This proposal was presented in some detail eg at the Nottingham conference in July 2008, and at the Beijing LQG 09 conference.
} 
a quite natural modification of the propagator $[3,4,5]$. This provides the scenario and main source of inspiration for our own views and hopes about quantum gravity. We will recall some key points of this story in the next section,

We will subsequently review the modest but already promising results that have been obtained on group field theory scaling and renormalization in the last two years.

\section{Scales}

In all quantum field theory models we know the renormalization group relies on three basic ingredients

- Scale decomposition,

- Locality principle,

- Power counting.

The theory is renormalizable when these three ingredients nicely fit together. Roughly speaking the key recipe is to relate the divergences of the theory to particular subgraphs whose internal scales are higher than their external scales. These graphs satisfy a locality principle. If by power counting their local parts keep the form of the terms already present in the action of the model, then the model is renormalizable. It means that the structure of the theory does not change with the observation scale, only the coupling constants move.

So right at the start lies the scale decomposition. It is perhaps both the most fundamental and the most technical ingredient. A scale decomposition can indeed be realized in many different technical ways, which should ultimately be equivalent. For instance a scale decomposition can be created through block spinning or through the use of many different shapes of momentum cutoffs. At first sight scale seems forever linked to the notion of space and distance. Small scales correspond to the ultraviolet regime and to short distances; large scales correspond to the infrared regime and to large distances. The names of infrared and ultraviolet come from Fourier analysis, and electrodynamics. Small details are indeed detected by sending highly energetic probes that can read them thanks to their high momenta or short wave lengths. 
However a background-independent quantum theory of space-time for gravity should start without assuming any fixed background metric, and ideally not even any particular space-time topology. So the first puzzling question arises: how to define a background-independent notion of scale and a renormalization group flow in the absence of any ordinary metric, and even of any particular topology?

The problem lies in our deeply rooted and often subconscious identification of the notion of scale with that of distance. Fortunately we have some models at our disposal to disentangle that identification. In the models of condensed matter scale is not given by ordinary distance but by momentum distance to an extended singularity, the Fermi surface [2]. The GrosseWulkenhaar model is a pedagogical toy model which allows us to experiment much further with a renormalization group that mixes the usual notions of short and long distance scales.

Based on this experience in condensed matter and non commutative field theory we propose the following more abstract definition of scale:

Definition 1. A scale is a slice of eigenvalues of a propagator gently ${ }^{2}$ cut according to a geometric progression.

So we turned the problem into that of finding the right propagator with the right non-trivial spectrum for quantum gravity. The progress lies in the fact that such abstract propagators can exist and have non-trivial spectrum in absence of any space-time manifold.

\section{The Grosse-Wulkenhaar model}

The Grosse-Wulkenhaar $\phi^{\star 4}$ model on the Moyal space $R^{4}$ with $1 /\left(p^{2}+\Omega x^{2}\right)$ propagator is just renormalizable $[3,4]$. Its renormalization is very interesting, since all three main ingredients are subtly changed. More precisely the renormalization of that model relies on

- A new scale decomposition which mixes the traditional ultraviolet and infrared scales,

\footnotetext{
${ }^{2}$ The word gently does not look scientific but should not be suppressed. It means eg if the scale is defined through momentum cutoffs it is good to use smooth cutoffs rather than sharp ones in order to ensure the corresponding dual decays of the sliced propagator.
} 
- A new locality principle, called Moyality,

- A new power counting under which only regular ${ }^{3}$ graphs do diverge.

A big unexpected bonus is the existence of a non trivial fixed point [5] which makes that GW model essentially the prime and simplest candidate for a fully consistent four dimensional quantum field theory.

\subsection{The new scale decomposition}

The multiscale analysis relies on slicing the Grosse-Wulkenhaar propagator $1 /\left[p^{2}+\Omega^{2}(\tilde{x})^{2}\right]$. This propagator has parametric representation in $x$ space

$$
\begin{aligned}
C(x, y) & =\frac{\theta}{4 \Omega}\left(\frac{\Omega}{\pi \theta}\right) \int_{0}^{\infty} d \alpha e^{-\frac{\mu_{0}^{2} \theta}{4 \Omega} \alpha} \\
& \frac{1}{(\sinh \alpha)^{2}} \exp \left(-\frac{\Omega}{\theta \sinh \alpha}\|x-y\|^{2}-\frac{\Omega}{\theta} \tanh \frac{\alpha}{2}\left(\|x\|^{2}+\|y\|^{2}\right)\right)
\end{aligned}
$$

involving the Mehler kernel rather than the heat kernel.

A convenient slice decomposition is made through the parametric representation

$$
C^{i}(x, y)=\int_{M^{-2 i}}^{M^{-2(i-1)}} d \alpha \cdots \leqslant K M^{2 i} e^{-c_{1} M^{2 i}\|x-y\|^{2}-c_{2} M^{-2 i}\left(\|x\|^{2}+\|y\|^{2}\right)},
$$

where $M$ is a fixed number, namely the ratio of the geometric slicing progression. The corresponding scales correspond to a mixture of the ordinary ultraviolet and infrared notions [4].

\subsection{The Moyality principle}

The Moyal vertex in direct space is proportional to

$$
\begin{aligned}
& \int \prod_{i=1}^{4} d^{4} x^{i} \phi\left(x^{i}\right) \delta\left(x_{1}-x_{2}+x_{3}-x_{4}\right) \delta\left(x_{1}-x_{2}+x_{3}-x_{4}\right) \\
& \exp \left(2 \imath \theta^{-1}\left(x_{1} \wedge x_{2}+x_{3} \wedge x_{4}\right)\right) .
\end{aligned}
$$

\footnotetext{
${ }^{3} \mathrm{~A}$ regular ribbon graph is a planar graph with all external legs on the outer boundary.
} 


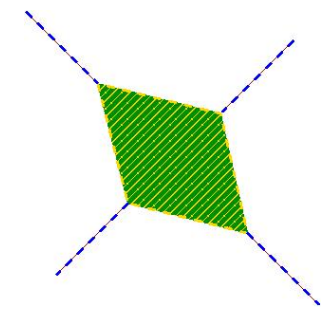

Figure 1: The Moyal vertex
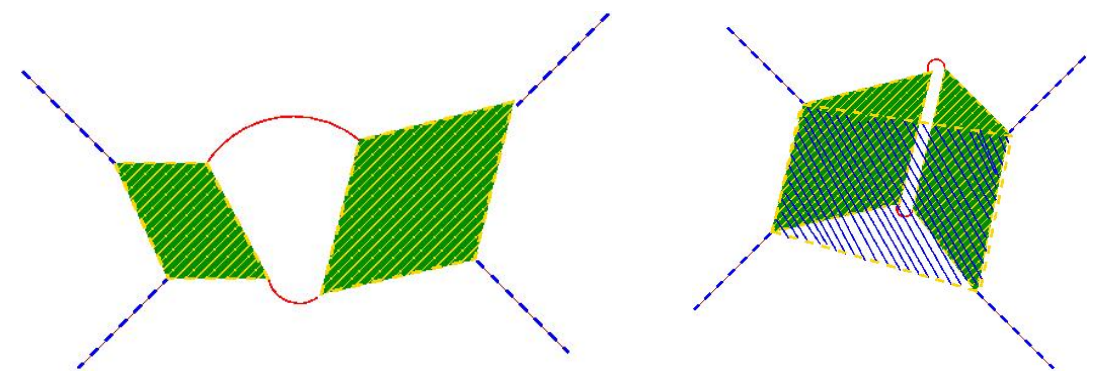

Figure 2: The Moyality principle

This vertex is non-local and oscillates. It has a parallelogram shape, and the phase of the oscillation is proportional to its area.

In Figure 2 it is shown how moving up the scales of the inner lines of a bubble glues its two parallelogram-shaped vertices into what from the external legs point of view appears as a new parallelogram, and how the inner oscillations add up to the correct one for the new parallelogram, since the area of the big parallelogram is the sum of the areas of the two smaller ones.

This Moyality principle applies only to regular, high subgraphs, where high means that all internal scales are higher than all external scales. Otherwise eg the oscillations won't add up correctly.

\subsection{Noncommutative Renormalization}

Renormalizability of the GW model combines the three elements. The new scale decomposition defines the "high" and "low" scales. The new power counting tells us that only regular graphs with two and four external legs must be renormalized. The Moyality principle says that such "high" regular graphs look like Moyal products. The corresponding counterterms are therefore of the form of the initial theory, thus the theory is renormalizable. 
An unexpected bonus is that it has a non-trivial ultraviolet fixed point [5]. Hence the theory should be fully consistent and non-trivial without cutoff. This is not expected to be the case of the ordinary $\phi_{4}^{4}$ theory, hence noncommutativity has indeed improved renormalizability but in a very subtle sense.

See [6] for further references on noncommutative field theory.

\section{Group Field Theory}

Group field theory (GFT) [7, 8] lies at the crossroads between the discretization of Cartan's first order formalism by loop quantum gravity, the higher dimensional tensorial extension of the matrix models relevant for 2D quantum gravity and the "Regge" or simplicial approach to quantum gravity

There are several enticing factors about GFT. The spin foams of loop gravity are exactly the Feynman amplitudes of group field theory [1]. However spin foam formalism alone still misses what we feel is a key ingredient, namely how to weight correctly different topologies of space time. That's why the spin foam formalism alone is still an incomplete proposal to quantize gravity: the amplitudes are there, but not the correct weights to sum them up. In contrast, GFT is an attempt to quantize completely space-time, summing at the same time over metrics and topologies. It assumes only the space-time dimension and does not privilege any manifold at the start. It could provide a scenario for emergent large, manifold-like classical space-time as a condensed phase of more elementary space-time quanta. If that is the case, there is no reason that some GFT's cannot hopefully be renormalized through a suitably adapted multiscale RG.

The main objections to GFT's that I heard during the last two years were

1. The GFT's are tensor generalizations of matrix models; but whereas matrix models really triangulate 2D Riemann surfaces, GFT's triangulate much more singular objects (not even pseudo-manifolds with local singularities).

2. There is no analog of the famous $1 / \mathrm{N}$ expansion for group field theories.

3. The natural GFT's action does not look positive. Hence the nonperturbative meaning of the theory is unclear. 
4. It seems difficult to identify ordinary space-time and physical observables in GFT's.

5. GFT's have infinities and it is not clear whether these infinities should or can be absorbed in a renormalization process;

6. GFT's don't predict space-time dimension, nor the standard model.

However at least some of the objections have been partly answered or are under detailed investigation. The first two items have been more or less already solved through the invention of colored group field theory $[9,10,11$, 12]. The third one may also be solved if, as colored field theory suggests, the GFT fields were eg Fermionic [9].

Concerning the fourth item, one could say that to identify ordinary spacetime and physical observables in GFT's is a non-trivial task, just as identifying hadrons out of quarks and gluons is. In particular the ultraspin limit $j \rightarrow \infty$ can be interpreted either as ultraviolet limit (on the group) or as an infrared limit for a large "chunk" of space-time. Quantum gravity might therefore be a theory with some kind of ultraviolet/infrared mixing, more complicated than in the GW model. However once large, topologically trivial $4 \mathrm{~d}$ space time is obtained as an effective phase of the GFT, general relativity on it should follow naturally if we have respected the principle of background independence, which should then imply diffeomorphism invariance of the theory on any preferred specific manifold.

Concerning the fifth item it is exactly the point that our program would like to investigate in detail. Progress has been made essentially for ordinary topological group field theories of the BF type and it is too early to draw conclusions for more general theories, that could well turn out to be just renormalizable with a suitable choice of the propagator.

Finally it is true that GFT's don't predict space-time dimension, nor the standard model, but the issue of completing GFT with matter fields is making progress $[14,15,16]$, and it is not clear to us whether the competition really fares any better at this point.

\subsection{GFT as theories of Holonomies}

Consider a manifold $M$ with a connection $\Gamma$ (eg Levi-Civita of a metric). The geometric information is encoded in the holonomies along all closed curves $\gamma$ in $M$. 
If $X$ is the vector field solution of the parallel transport equation

$$
\frac{d \gamma^{\nu}}{d t} \partial_{\nu} X^{\mu}+\Gamma_{\nu \sigma}^{\mu} X^{\nu} \frac{d \gamma^{\sigma}}{d t}=0, \quad X(0)=X_{0}
$$

then $X(T)=g X_{0}$ for some $g \in G L\left(T M_{\gamma(0)}\right) . g$ (independent of $X_{0}$ ) is the holonomy along the curve $\gamma$. Suppose we discretize $M$ with flat $n$ dimensional simplices. Their boundary $(n-1$ dimensional) is also flat. The curvature is located at the "joints" of these blocks, that is at $n-2$ dimensional cells. Hence holonomies $h$ are associated to blocks of codimension 2 .

\section{2 $2 D$ GFT as Matrix Models}

Consider a two dimensional surface $M$, and fix a triangulation of $M$. The holonomy group of a surface is $G=U(1)$. To all vertices (points) in our triangulation we associate a holonomy $g$, encoding the deficit angle along any small curve encircling the vertex. The surface and its metric are specified by the gluing of the triangles and by the holonomies $g$. We associate to this

surface a weight function $F\left(g, g^{\prime}, \ldots\right)$. Quantization of geometry should sum both over metrics compatible with a triangulation and over all triangulations.

The discrete information about the triangulation of the surface can be encoded in a ribbon (2-stranded) dual graph. The ribbon vertices of the graph are dual to triangles and the ribbon lines are dual to edges.

In the dual graph the group elements $g$ are associated to the sides of the ribbons, also called strands. Suppose that the weight function $F$ factors into contributions of dual vertices and dual lines:

$$
F=\prod_{V} V\left(g_{1}, g_{2}, g_{3}\right) \prod_{L} K\left(g_{1}, g_{2}\right)
$$

Then a 2-stranded graph is a ribbon Feynman graph (and its weight is the integrand of the Feynman amplitude) of a matrix model defined by the action

$$
\begin{aligned}
& S=\frac{1}{2} \int_{G \times G} \phi\left(g_{1}, g_{2}\right) K^{-1}\left(g_{1}, g_{2}\right) \phi^{*}\left(g_{1}, g_{2}\right) \\
& +\lambda \int_{G \times G \times G} V\left(g_{1}, g_{2}, g_{3}\right) \phi\left(g_{1}, g_{2}\right) \phi\left(g_{2}, g_{3}\right) \phi\left(g_{3}, g_{1}\right),
\end{aligned}
$$

with $\phi\left(g_{1}, g_{2}\right)=\phi^{*}\left(g_{2}, g_{1}\right)$. Taking $K, V=1$ and developing $\phi$ in Fourier series

$$
\phi\left(g_{1}, g_{2}\right)=\sum_{\mathbb{Z} \times \mathbb{Z}} e^{\imath m g_{1}} e^{-\imath n g_{2}} \phi_{m n}
$$


the action takes the more familiar form

$$
S=\frac{1}{2} \sum_{m n} \phi_{m n} \phi_{m n}^{*}+\lambda \sum_{m n k} \phi_{m n} \phi_{n k} \phi_{k m} .
$$

A complete correlation function of a matrix model

$$
<\phi\left(g_{1}, g_{2}\right) \ldots \phi\left(g_{2 n-1}, g_{2 n}\right)>=\int[d \phi] e^{-S} \phi\left(g_{1}, g_{2}\right) \ldots \phi\left(g_{2 n-1}, g_{2 n}\right)
$$

admits the following interpretation. The insertions $\phi\left(g_{1}, g_{2}\right)$ fix a boundary triangulation and geometry. Each Feynman graph fixes a bulk triangulation. The amplitude of a graph is the sum over all bulk geometries compatible with the triangulation. The correlation function automatically sums the weights of all triangulations and geometries compatible with the boundary! As such the matrix models provide a quantization of 2D pure gravity.

\subsection{General Relativity in three dimensions}

In Cartan's formalism, 3D quantum gravity is expressed in terms of a dreibein

$$
e^{i}(x)=e_{a}^{i}(x) d x^{a}
$$

and a spin connection $\omega$ with values in the $s o(3)$ Lie algebra

$$
\omega^{i}(x)=\omega_{a}^{i}(x) d x^{a} .
$$

The action is

$$
S(e, \omega)=\int e_{i} \wedge F(\omega)^{i}
$$

where $F$ is the curvature of $\omega$ :

$$
F(\omega)=d \omega+\omega \wedge \omega
$$

Varying $\omega$ gives Cartan's equation $D e=0$. Varying $e$ gives $F=0$, hence a flat space-time. 3D gravity is a topological theory with only global observables and no propagating degrees of freedom. Nevertheless the theory is physically interesting. Matter can be added; point particles do not curve space but induce an angular deficit proportional to their mass. Therefore in $3 \mathrm{D}$ gravity there is a limit $(2 \pi)$ to any point-particle's mass. 


\subsection{Group Field Theory in three dimensions}

In three dimensions we must use the holonomy group $G=S U(2)$, the universal covering of the $S O(3)$ rotations group. Group elements are associated to edges in the triangulation (codimension 2). Each field $\phi$ is associated to a triangular face of a tetrahedron, therefore it has three arguments. The propagator $K$ is the inverse of the quadratic part

$$
\frac{1}{2} \int_{G^{3}} \phi\left(g_{a}, g_{b}, g_{c}\right) K^{-1}\left(g_{a}, g_{b}, g_{c}\right) \phi^{*}\left(g_{a}, g_{b}, g_{c}\right) \text {. }
$$

This vertex is dual to a tetrahedron. A tetrahedron is bounded by four triangles therefore the vertex is a $\phi^{4}$ term, namely

$$
\lambda \int_{G^{6}} V(g, \ldots, g) \phi\left(g_{03}, g_{02}, g_{01}\right) \phi\left(g_{01}, g_{13}, g_{12}\right) \phi\left(g_{12}, g_{02}, g_{23}\right) \phi\left(g_{23}, g_{13}, g_{03}\right) \text {. }
$$

What characterizes vertices in QFT is some kind of locality property. Similarly we propose

Definition 2. A vertex joining $2 p$ strands in GFT is called simple if it has for kernel in direct group space a product of $p$ delta functions matching strands two by two in different half-lines.

The natural 3D GFT tetrahedron vertex in 3 dimensions is simple in this sense (with $p=6$ ) as it writes

$$
\begin{array}{r}
V[\phi]=\lambda \int\left(\prod_{i=1}^{12} d g_{i}\right) \phi\left(g_{1}, g_{2}, g_{3}\right) \phi\left(g_{4}, g_{5}, g_{6}\right) \\
\phi\left(g_{7}, g_{8}, g_{9}\right) \phi\left(g_{10}, g_{11}, g_{12}\right) Q\left(g_{1}, . . g_{12}\right),
\end{array}
$$

with a kernel

$$
Q\left(g_{1}, . . g_{12}\right)=\delta\left(g_{3} g_{4}^{-1}\right) \delta\left(g_{2} g_{8}^{-1}\right) \delta\left(g_{6} g_{7}^{-1}\right) \delta\left(g_{9} g_{10}^{-1}\right) \delta\left(g_{5} g_{11}^{-1}\right) \delta\left(g_{1} g_{12}^{-1}\right) .
$$

The 3D GFT propagator should implement the flatness condition. The propagator

$$
[C \phi]\left(g_{1}, g_{2}, g_{3}\right)=\int d h \phi\left(h g_{1}, h g_{2}, h g_{3}\right)
$$

is a projector onto gauge invariant fields $\phi\left(h g, h g^{\prime}, h g^{\prime \prime}\right)=\phi\left(g, g^{\prime}, g^{\prime \prime}\right)$. It has kernel

$$
C=\int d h \delta\left(g_{1} h g_{1}^{\prime-1}\right) \delta\left(g_{2} h g_{2}^{\prime-1}\right) \delta\left(g_{3} h g_{3}^{\prime-1}\right)
$$


Performing the integrations over all group elements of the vertices and keeping the $h$ elements unintegrated gives for each triangulation, or 3-stranded graph $G$ a Feynman amplitude

$$
Z_{G}=\int \prod_{e} d h_{e} \prod_{f} \delta\left(g_{f}\right)
$$

where $g_{f}=\vec{\prod}_{e \in f} h_{e}$ is the holonomy along the face $f$. Therefore 3D GFT with the projector $C$ as propagator implements exactly the correct flatness conditions of 3D gravity [13]! Fourier transforming the model, one gets PonzanoRegge amplitudes.

This 3D GFT is topological and the amplitude of a graph changes through a global multiplicative factor under the so-called Pachner moves. Amplitudes may be infinite, for instance the tetrahedron graph or complete graph $K_{4}$ diverges as $\Lambda^{3}$ at zero external data, if $\Lambda$ is the ultraviolet cutoff on the size of $j$ 's. Regularization by going to a quantum group at a root of unity leads to well-defined topological invariants of the triangulation, namely the TuraevViro invariants. However the theory seems unsuited for a true RG analysis, as the propagator has spectrum limited to 0 and 1 . It cannot therefore be used to define truly scales in the abstract sense of Definition 1.

\subsection{The 4 Dimensional Case}

In the first order Cartan formalism, the action is proportional to $\int \star[e \wedge$ $e] \wedge F$ where again the vierbein $e$ and the spin connection $\omega$ are considered independent variables.

An approach to gravity in 4 dimensions is to consider it as a constrained $B F$ theory. The $B F$ theory, of action $\int B \wedge F$, involves an arbitrary two form $B$. As not all two-forms $B$ in four dimenions are of the form $e \wedge e$, one needs to implement a certain number of constraints on the $B$ field, the so called Plebanski constraints. These constraints render 4D gravity much more complicated and interesting since they are responsible for the local propagating degrees of freedom, the gravitational waves, as constraints on $B$ allow richer set of $F$ 's than just $F=0$.

Still another action classically equivalent to the Einstein-Hilbert action is the Holst action:

$$
S=-\frac{1}{8 \pi G} \int\left[{ }^{\star}(e \wedge e)+\frac{1}{\gamma}(e \wedge e)\right] \wedge F
$$




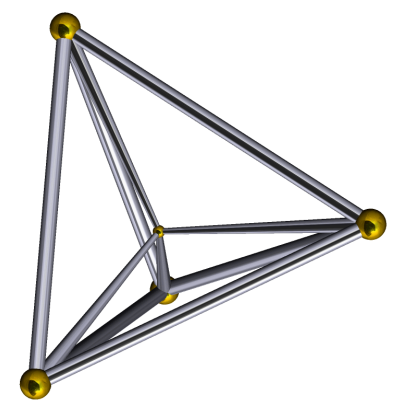

Figure 3: The pentachore

where the first term is the Palatini action, and the second one (the Holst term) is topological. The parameter $\gamma$ (called the Barbero-Immirzi parameter) plays no rôle at the classical level but is crucial to the loop quantization of $4 \mathrm{D}$ gravity [1]. We can then rewrite this action à la Plebanski (i.e. $B F$ theory plus constraints) and get the Palatini-Holst-Plebanski functional integral measure, loosely written as:

$$
d \nu=\frac{1}{Z} e^{-\frac{1}{8 \pi G} \int\left[{ }^{\star} B+\frac{1}{\gamma} B\right] \wedge F} \delta(B=e \wedge e) D B D \omega .
$$

\subsubsection{D GFT Vertex}

The vertex is the easy part as it should be again given by gluing rules for the five tetrahedra which join into pentachores.

The corresponding graphs are made of vertices dual to the pentachores, of four-stranded edges dual to tetrahedra, and of faces or closed thread circuits dual to triangles. If we keep the same propagator than in the Boulatov theory

$$
[C \phi]\left(g_{1}, g_{2}, g_{3}, g_{4}\right)=\int d h \phi\left(h g_{1}, h g_{2}, h g_{3}, h g_{4}\right)
$$

we get a $\phi^{5}$ GFT with 4-stranded graphs called the Ooguri group field theory [17]. It is not $4 \mathrm{D}$ gravity, but a discretization of the $4 \mathrm{D} \mathrm{BF}$ theory, ie the Plebanski constraints are missing. Again such a topological version of gravity is unsuited for RG analysis.

A first attempt to implement the Plebanski constraints in this language is due to Barrett and Crane. They suggested one should restrict to simple 
representations of $S U(2) \times S U(2)$, namely those satisfying $j_{+}=j_{-}$. However this does not seem to work because the Barrett-Crane proposal may implement the Plebanski constraints too strongly. In particular it may not lead to the correct angular degrees of freedom of the graviton.

\section{The EPR/FK theory}

Parallel works by Engle-Pereira-Rovelli and by Freidel-Krasnov, with contributions of Livine and Speziale, lead in 2007 to an improved spin foam model $[18,19,20]$ which may better incorporate the Plebanski constraints and the angular degrees of freedom of the graviton. It translates into an new proposal for a $4 \mathrm{D}$ GFT [21] with an improved propagator which incorporates

- A $S U(2) \times S U(2)$ averaging $C$ at both ends like in Ooguri theory

- A simplicity constraint which depends on the value of $\gamma$ :

$$
j_{+}=\frac{1+\gamma}{2} j ; \quad j_{-}=\frac{1-\gamma}{2} j \text { for } 0<\gamma<1
$$

- A new projector $S$ averaging on a single $S U(2)$ residual gauge invariance in the middle of the propagator.

\subsection{Renormalization group, at last?}

The main new feature of the EPR/FK models is that the full propagator can therefore be written as $K=C S C$ with $C^{2}=C$ and $S^{2}=S$, hence $C$ and $S$ are two projectors, but they do not commute, hence $K$ has non-trivial spectrum! This model is therefore a natural starting point for a $R G$ analysis along our program.

A first step in this direction has been performed in 2008 [22]. In some normalization of the theory a tantalizing logarithmic divergence for a radiative correction to the coupling constant and a $\Lambda^{6}$ divergence for the graph $G_{2}$

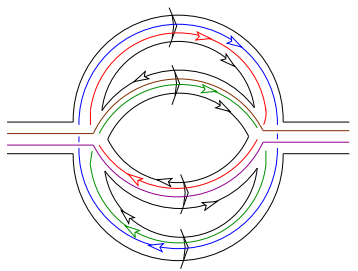


have been found.

\subsection{What has been done so far}

${ }^{4}$ We have investigated the power counting of the Boulatov model, and found the first uniform bounds for its Freidel-Louapre constructive regularization, using the loop vertex expansion technique [23].

We have computed in the Abelian case the power counting of graphs for $B F$ theory, and found how to relate them to the number of bubbles of the graphs in the colored case, along the lines of $[26,9]$. The Abelian amplitudes lead to new class of topological polynomials for stranded graphs [24, 25]. See also the related work of Bonzom and Smerlak [27, 28] and [29] for colored polynomials.

We have started the study of the general superficial degree of divergence of EPR/FK graphs through saddle point analysis using coherent states techniques [30]. We recover in many cases the Abelian counting, and find also corrections to it in general. But saddle points may come in many varieties, degenerate or not. This richness is interesting but a major challenge. We recovered the $\Lambda^{6}$ divergence of the EPR/FK graph $G_{2}$ in the case of non-degenerate saddle point configurations, but also a $\Lambda^{9}$ divergence for the maximally degenerate saddle points [30]. Our method also works at non zero external spins .

We have written still a more compact representation of the GFT with EPR/FK propagator in terms of traces [21].

Finally let us mention recent results which establish the $1 / \mathrm{N}$ expansion for colored models and prove that spheres dominate in all dimensions [11, $12,31]$. This is encouraging for generating a large topologically trivial world as we know it. In colored theory the issue of respecting suitable discretized diffeomorphism invariance seems also more promising [32].

\subsection{What remains to be done}

Essentially everything!

- We need to establish results such as the dominance of spherical graphs not only in the BF but also in the EPR/FK colored models. The jacket

\footnotetext{
${ }^{4}$ This section mostly summarizes joint work with J. Ben Geloun, R. Gurau, T. Krajewski, J. Magnen, K. Noui, M. Smerlak, A. Tanasa and P. Vitale.
} 
bounds of the $1 / N$ expansion $[11,12,31]$ seem a promising starting point in this direction.

- We need to cut propagators with non trivial spectra like the EPR/FK into slices according to their spectrum. Such a slice requires two cutoffs, one for the top one for the bottom of the slice. Then we can perform a true multiscale RG analysis, study the "high" subgraphs, which should obey the new locality principle, and establish their correct power counting. Only then can we find whether some of these models are renormalizable or not.

- We need to find out whether symmetries such as the tantalizing topological $B F$ symmetry recovered at $\gamma=1$ create fixed points of the RG analysis.

- We need to get a better glimpse of whether a phase transition to a condensed phase can lead to emergence of a large smooth space-time.

\section{Conclusion and further Outlook}

Let us warn that this section is of a purely speculative nature and that of course none of my collaborators are responsible for these speculations which will turn out to be wrong!

Suppose some group field theory with the simple pentachore vertex and a yet to be specified propagator turns out to be renormalizable with an ultraviolet fixed point and a low energy phase transition leading to the ordinary effective world of general relativity on a four dimensional space-time. What would it mean for physics?

The physics of the universe from far "before" the big bang to the current cosmos could be summarized as a single giant renormalization group ( $R G$ ) trajectory. It would flow out from very near a simple very high or infinitely high "transplanckian" fixed point ${ }^{5}$. This fixed point might be characterized by the value 1 of the Immirzi parameter and might be topological in nature. Our world could then be called asymptotically topological, just like

\footnotetext{
${ }^{5}$ Of course the next mystery would be to understand the vicinity of this fixed point and why the world emerged at all out of it...
} 
Yang-Mills theory is asymptotically free. We hope the vicinity of that transplanckian fixed point could be fully understood with analytical tools which do not require computer simulations, like is the case for integrable systems and probably for the Grosse-Wulkenhaar fixed point [34]. Cooling to the big bang and below, the RG trajectory of the universe could undergo many phase transitions until our large and smooth world appears to us as it is today. The emergence of space time, called geometrogenesis, would occur through a condensation of the vertices of group field theory into a non perturbative phase. Through later transitions space-time cools further and becomes filled with the particles and fields of the standard model at small scales and with the galaxies we actually observe at large scales. It is only after the geometrogenesis that we can really distinguish between these large infrared and small ultraviolet scales in the traditional sense; before geometrogenesis there are only the abstract "ultraspin" scales. Ordinary distances cannot be measured and time itself should be replaced by this abstract notion of "scale". We could rather imagine the world as made of loosely interacting pentachores, sort of atoms of space time that would float in an abstract vacuum which is no space-time at all. These atoms have not condensed yet, that is they are not bound into any specific manifold.

This transplanckian era of the universe would be characterized by the flow of the Immirzi parameter from 1 to some non trivial value. Slightly below the Planck scale and geometrogenesis, semiclassical computations involving classical geometry coupled to quantized matter, such as the computation of the Bekenstein-Hawking radiation, would begin to make sense, as macroscopic areas and horizons start to appear. Therefore the Immirzi parameter, intrinsically quantum geometric, could freeze there, at a value which we might fix so as to recover the usual Bekenstein-Hawking entropy. This would give a renormalized effective value for $\gamma$ which in loop quantum gravity is usually considered to be close to $\log 3 / \pi \sqrt{2}[33]$.

However summarizing the transplanckian physics as a simple flow of $\gamma$ would not be quite right yet. We also expect the coupling constant $\lambda$ of the group field theory vertex to move, and probably a few other operators as well, which might be related to the future matter and radiation fields of the standard model. In the model case of the Grosse-Wulkenhaar model, fixing the wave function factor in front of the $p^{2}$ term to 1 is a way of fixing the energy scales of the renormalization group. Then two other parameters have non-trivial logarithmic RG flows, namely $\Omega$, the harmonic potential term which measures the rate of mixing of infrared and ultraviolet, and the 
coupling constant $\lambda$. At $\Omega=1$, hence perfect mixing, the beta function vanishes because of the enhanced symmetry [5]. By analogy we expect also both $\gamma$ and $\lambda$ to flow from a fixed point at $\gamma=1$, at which the beta function should vanish because of the purely topological nature of the GFT at that point.

Running the RG flow from that $\gamma=1$ fixed point towards our effective world, the coupling constant might grow from a small value to a larger value where a non-perturbative phase transition occurs. This is what happens in the BCS theory of superconductivity or in QCD. For this condensation to occur and some manifold-like space time to emerge ${ }^{6}$, two main things are required. First perturbation theory should be at the verge of diverging so that pentachores should pullulate; second they should energetically prefer to glue in a nice and coherent pattern so as to create a smooth manifold, say a sphere. Both phenomena should occur when $\lambda$ reaches a critical value $\lambda_{c}$ at which the sum of all "spherical graphs" would diverge. A spherical graph is the analog of a planar graph in matrix models. Just like planar graphs dominate in power counting in matrix and in Grosse-Wulkenhaar models, spherical graphs dominate in colored 4D GFTs [12]. Just like the asymptotic behavior of the planar series at large order $n$ is in $K_{P}^{n}$ we expect the asymptotic behavior of these spherical graphs to be in $K_{S}^{n}$ at large order, with an analytically computable value of $K_{S}$. The value of $\lambda_{c}$ should simply be $1 / K_{S}$.

The vicinity of the phase transition being around $\lambda=\lambda_{c}$ might perhaps be probed non perturbatively with a double scaling limit. In any case this phase transition should be more complex than BCS condensation which is vector like, or quark confinement which is matrix like; it is " $4 \mathrm{~d}$-manifoldlike" hence a new step in complexity. This part of the scenario is quite murky and it may be that for some time we have to investigate it through computer simulations, just like quark confinement and hadron formation are still studied today.

Acknowledgments This review benefitted from discussions with many people. Let me thank more particularly Razvan Gurau and Jacques Magnen, and also Joseph Ben Geloun, Harald Grosse, Thomas Krajewski, Karim Noui, Daniele Oriti, Carlo Rovelli, Matteo Smerlak, Simone Speziale, Adrian

\footnotetext{
${ }^{6}$ We would like also this manifold or at least its main four-dimensional part to be topologically trivial, i.e. spherical, because our four dimesnional universe does not look full of macroscopic holes or handles.
} 
Tanasa, Patrizia Vitale and Raimar Wulkenhaar.

\section{References}

[1] C. Rovelli, Quantum Gravity, Cambridge University Press (2004).

[2] V. Rivasseau, "Introduction to the Renormalization Group with Applications to Non-Relativistic Quantum Electron Gases," arXiv:1102.5117 [math-ph].

[3] H. Grosse and R. Wulkenhaar, "Renormalisation of phi**4 theory on noncommutative $\mathrm{R}^{* *} 4$ in the matrix base," Commun. Math. Phys. 256, 305 (2005) [arXiv:hep-th/0401128].

[4] R. Gurau, J. Magnen, V. Rivasseau and F. Vignes-Tourneret, "Renormalization of non-commutative phi**4(4) field theory in x space," Commun. Math. Phys. 267, 515 (2006) [arXiv:hep-th/0512271].

[5] M. Disertori, R. Gurau, J. Magnen and V. Rivasseau, "Vanishing of beta function of non commutative phi $(4)^{* *} 4$ theory to all orders," Phys. Lett. B 649, 95 (2007) [arXiv:hep-th/0612251].

[6] V. Rivasseau, "Non-commutative renormalization," arXiv:0705.0705 [hep-th].

[7] L. Freidel, "Group field theory: An overview," Int. J. Theor. Phys. 44, 1769 (2005) [arXiv:hep-th/0505016].

[8] D. Oriti, "The group field theory approach to quantum gravity: some recent results," [arXiv:0912.2441 [hep-th]].

[9] R. Gurau, "Colored Group Field Theory," [arXiv:0907.2582 [hep-th]].

[10] R. Gurau, "Lost in Translation: Topological Singularities in Group Field Theory," Class. Quant. Grav. 27, 235023 (2010) [arXiv:1006.0714 [hepth]].

[11] R. Gurau, "The 1/N expansion of colored tensor models," arXiv:1011.2726 [gr-qc]. 
[12] R. Gurau and V. Rivasseau, "The 1/N expansion of colored tensor models in arbitrary dimension," arXiv:1101.4182 [gr-qc].

[13] D. V. Boulatov, "A Model of three-dimensional lattice gravity," Mod. Phys. Lett. A 7, 1629 (1992) [arXiv:hep-th/9202074].

[14] W. J. Fairbairn and E. R. Livine, Class. Quant. Grav. 24, 5277 (2007) [arXiv:gr-qc/0702125].

[15] D. Oriti, "Emergent non-commutative matter fields from Group Field Theory models of quantum spacetime," J. Phys. Conf. Ser. 174, 012047 (2009) [arXiv:0903.3970 [hep-th]].

[16] A. Di Mare and D. Oriti, "Emergent matter from 3d generalised group field theories," Class. Quant. Grav. 27, 145006 (2010) [arXiv:1001.2702 [gr-qc]].

[17] H. Ooguri, "Topological lattice models in four-dimensions," Mod. Phys. Lett. A 7, 2799 (1992) [arXiv:hep-th/9205090].

[18] J. Engle, R. Pereira and C. Rovelli, "Flipped spinfoam vertex and loop gravity," Nucl. Phys. B 798, 251 (2008) [arXiv:0708.1236 [gr-qc]].

[19] L. Freidel and K. Krasnov, "A New Spin Foam Model for 4d Gravity," Class. Quant. Grav. 25, 125018 (2008) [arXiv:0708.1595 [gr-qc]].

[20] E. R. Livine and S. Speziale, "A new spinfoam vertex for quantum gravity," Phys. Rev. D 76, 084028 (2007) [arXiv:0705.0674 [gr-qc]].

[21] J. B. Geloun, R. Gurau and V. Rivasseau, "EPRL/FK Group Field Theory," Europhys. Lett. 92, 60008 (2010) [arXiv:1008.0354 [hep-th]].

[22] C. Perini, C. Rovelli and S. Speziale, "Self-energy and vertex radiative corrections in LQG," Phys. Lett. B 682, 78 (2009) [arXiv:0810.1714 [gr-qc]].

[23] J. Magnen, K. Noui, V. Rivasseau and M. Smerlak, "Scaling behaviour of three-dimensional group field theory," Class. Quant. Grav. 26, 185012 (2009) [arXiv:0906.5477 [hep-th]]. 
[24] J. B. Geloun, J. Magnen and V. Rivasseau, "Bosonic Colored Group Field Theory," Eur. Phys. J. C 70, 1119 (2010) [arXiv:0911.1719 [hepth]].

[25] J. B. Geloun, T. Krajewski, J. Magnen and V. Rivasseau, "Linearized Group Field Theory and Power Counting Theorems," Class. Quant. Grav. 27, 155012 (2010) [arXiv:1002.3592 [hep-th]].

[26] L. Freidel, R. Gurau and D. Oriti, "Group field theory renormalization - the 3d case: power counting of divergences," Phys. Rev. D 80, 044007 (2009) [arXiv:0905.3772 [hep-th]].

[27] V. Bonzom and M. Smerlak, "Bubble divergences from cellular cohomology," Lett. Math. Phys. 93, 295 (2010) [arXiv:1004.5196 [gr-qc]].

[28] V. Bonzom and M. Smerlak, "Bubble divergences from twisted cohomology," arXiv:1008.1476 [math-ph].

[29] R. Gurau, "Topological Graph Polynomials in Colored Group Field Theory," Annales Henri Poincare 11, 565 (2010) [arXiv:0911.1945 [hep-th]].

[30] T. Krajewski, J. Magnen, V. Rivasseau, A. Tanasa and P. Vitale, "Quantum Corrections in the Group Field Theory Formulation of the EPRL/FK Models," Phys. Rev. D 82, 124069 (2010) [arXiv:1007.3150 [gr-qc]].

[31] R. Gurau, "The complete $1 / \mathrm{N}$ expansion of colored tensor models in arbitrary dimension," arXiv:1102.5759 [gr-qc].

[32] A. Baratin, F. Girelli and D. Oriti, "Diffeomorphisms in group field theories," arXiv:1101.0590 [hep-th].

[33] O. Dreyer "Quasinormal Modes, the Area Spectrum, and Black Hole Entropy", Phys. Rev. Lett. w90, 081301 (2003).

[34] H. Grosse and R. Wulkenhaar, "Progress in solving a noncommutative quantum field theory in four dimensions," arXiv:0909.1389 [hep-th]. 